\title{
External Validation of the Core Obesity Model to Assess the Cost-Effectiveness of Weight Management Interventions
}

\author{
Sandra Lopes $^{1} \cdot$ Pierre Johansen $^{1}\left[\right.$ Mark Lamotte $^{2} \cdot$ Phil McEwan $^{3}$ (C) $\cdot$ Anamaria-Vera Olivieri $^{4} \cdot$ Volker Foos $^{3}$
}

Published online: 13 July 2020

(c) The Author(s) 2020

\begin{abstract}
Background For economic models to be considered fit for purpose, it is vital that their outputs can be interpreted with confidence by clinicians, budget holders and other stakeholders. Consequently, thorough validation of models should be carried out to enhance confidence in their predictions. Here, we present results of external dependent and independent validations of the Core Obesity Model (COM), which was developed to assess the cost-effectiveness of weight management interventions. Objective The aim was to assess the external validity of the COM (version 6.1), in line with best practice guidance from the International Society for Pharmacoeconomics and Outcomes Research and the Society for Medical Decision Making.

Methods For validation, suitable sources and outcomes were identified, and used to populate the COM with relevant inputs to allow prediction of study outcomes. Study characteristics were entered into the COM to replicate either the studies used to develop the model (dependent validation) or those not included in the model (independent validation). The concordance between predicted and observed outcomes was then assessed using established statistical methods and generation of mean error estimates.

Results For most outcomes, the predictions of the COM showed good linear correlation with observed outcomes, as evidenced by the high coefficients of determination ( $R^{2}$ values). The independent validation revealed a degree of underestimation in predictions of cardiovascular (CV) disease and mortality, and type 2 diabetes.

Conclusion The predictions generated by the risk equations used in the COM showed good concordance both with the studies used to develop the model and with studies not included in the model. In particular, the concordance observed in the external dependent validation suggests that the COM accurately predicts obesity-related event rates observed in the studies used to develop the model. However, the impact of existing CV risk, as well as mortality, is a key area for future refinement of the COM. Our results should increase confidence in the estimates derived from the COM and reduce uncertainty associated with analyses using this model.
\end{abstract}

\section{Introduction}

Obesity, defined as a body mass index (BMI) of $30 \mathrm{~kg} / \mathrm{m}^{2}$ or greater, affects more than 650 million people worldwide [1], and is increasing in prevalence. The clinical, economic and humanistic impact of obesity is considerable, in large

Electronic supplementary material The online version of this article (https://doi.org/10.1007/s40273-020-00941-3) contains supplementary material, which is available to authorized users.

Sandra Lopes

snlb@novonordisk.com

Novo Nordisk A/S, Søborg, Denmark

IQVIA, Zaventem, Belgium

Health Economics and Outcomes Research Ltd, Cardiff, UK

IQVIA, Basel, Switzerland

\section{Key Points for Decision Makers}

Validation of economic model accuracy is essential to ensure confidence in the outcomes generated.

We applied best practice guidelines to perform external validation of how accurately the Core Obesity Model (COM) predicted rates of obesity-related complications, including cardiovascular disease, type 2 diabetes, obstructive sleep apnoea and mortality.

The COM predictions show good concordance with the results of studies used to develop the model and those not included in the model, suggesting that the model is suitable for decision-making. 
part due to the multifactorial aetiology and chronic nature of complications. Known complications linked to obesity comprise obstructive sleep apnoea (OSA) [2], type 2 diabetes (T2D), hypertension, dyslipidaemia and cardiovascular disease (CVD), including stroke, myocardial infarction (MI) and coronary heart disease [3, 4]; consequently, obesity is associated with severe long-term morbidity and increased mortality. The impact of obesity on health-related quality of life (HRQoL) is also well documented. It is well established that obesity has a psychological impact as a result of social stigmatization, exclusion and isolation [5]; this finding is supported by data indicating that obesity is associated with significantly reduced scores for both the physical and mental components of the 36-item Short-Form Health Survey (SF-36) [6].

Obesity is associated with high direct costs to healthcare systems [7], a large proportion of which arise from treating comorbidities [8], as well as substantial indirect costs to society [9]. Given the high prevalence of obesity and its comorbidities, the development of economic models is vital for providing long-term estimates of its clinical effects and economic impact, allowing comparison of weight management interventions and informing healthcare decisions about allocation of scarce resources. The evidence used for these decisions is often taken from clinical trials, which typically assess outcomes over short-term follow-up periods; however, in many cases, certain treatment benefits may be detected only several years, or even decades, after initiation of therapy [10]. Therefore, the use of economic models based on short-term clinical data to project long-term outcomes is widely used to aid decision-making [11].

The usefulness of an economic model depends on it being fit for purpose; furthermore, it is vital that its outputs can be interpreted with confidence by clinicians, budget holders and other stakeholders. This need for accuracy and transparency in model development has led to the development of best practice guidelines by, for example, the International Society for Pharmacoeconomics and Outcomes Research (ISPOR) and the Society for Medical Decision Making (SMDM) [12, 13]. For $\mathrm{T} 2 \mathrm{D}$, validation of existing health economic models has been carried out widely and according to best-practice guidance, with collaboration between study groups to assess and compare different models $[14,15]$. In contrast, although some obesity models have been the subject of validation studies, formal validation has not been attempted for a large proportion of published models. In a systematic review of obesity models, only $14 \%$ (10/72) of the identified modelbased health economic assessments included a published external validation, highlighting a general lack of such validations [16].

The Core Obesity Model (COM) is a Markov, closedcohort model consisting of health states that represent the most common complications associated with obesity (Fig. 1)
[17]. The inputs can be adjusted to model populations with normal weight, overweight or obesity, allowing comparison of outcome risks for different weight profiles. The outcomes included in the model are considered to have either strong evidence (T2D, acute coronary syndrome [ACS], stroke and sleep apnoea) or moderate evidence (knee replacement, postmenopausal endometrial cancer, postmenopausal breast cancer and colorectal cancer) for their association with obesity, based on a comprehensive report from the World Health Organization [18]. Furthermore, these outcomes are affected by weight loss, and can have substantial consequences for healthcare resource use and costs, patients' quality of life and/or life expectancy. The COM also allows inclusion of healthcare payer, patient and societal costs, permitting costeffectiveness analyses encompassing the broad impact of obesity.

State-transition models have previously been used to model obesity [19-21] and diabetes [22], indicating that this approach is well suited to modelling a condition characterized by recurrent risks. Furthermore, by using a cohort statetransition model, we aimed to maximize transparency and user-friendliness while minimizing run-times and avoiding some common disadvantages of micro-simulation models relating to extensive model code and computational intensiveness [23].

The major focus of this study is external validation to determine how accurately the COM predicts outcomes strongly associated with obesity, including CVD (defined as ACS and stroke; heart failure and peripheral artery disease are not included among COM outcomes), T2D, obstructive sleep apnoea and mortality (cardiovascular $[\mathrm{CV}]$ and all-cause).

\section{Objectives}

In this study, we report the validation of the COM (version 6.1) in line with published guidance [13], with a focus on external validation of the model by comparison with results of published epidemiological and clinical studies, thus assessing whether the model is robust and fit for purpose.

\section{Methods}

The best practice guidelines from ISPOR/SMDM recommend five forms of validation: face validity, verification, cross validation, external validation and predictive validation [13]. The major focus of this study is on the results observed during the external (dependent and independent) validations. 


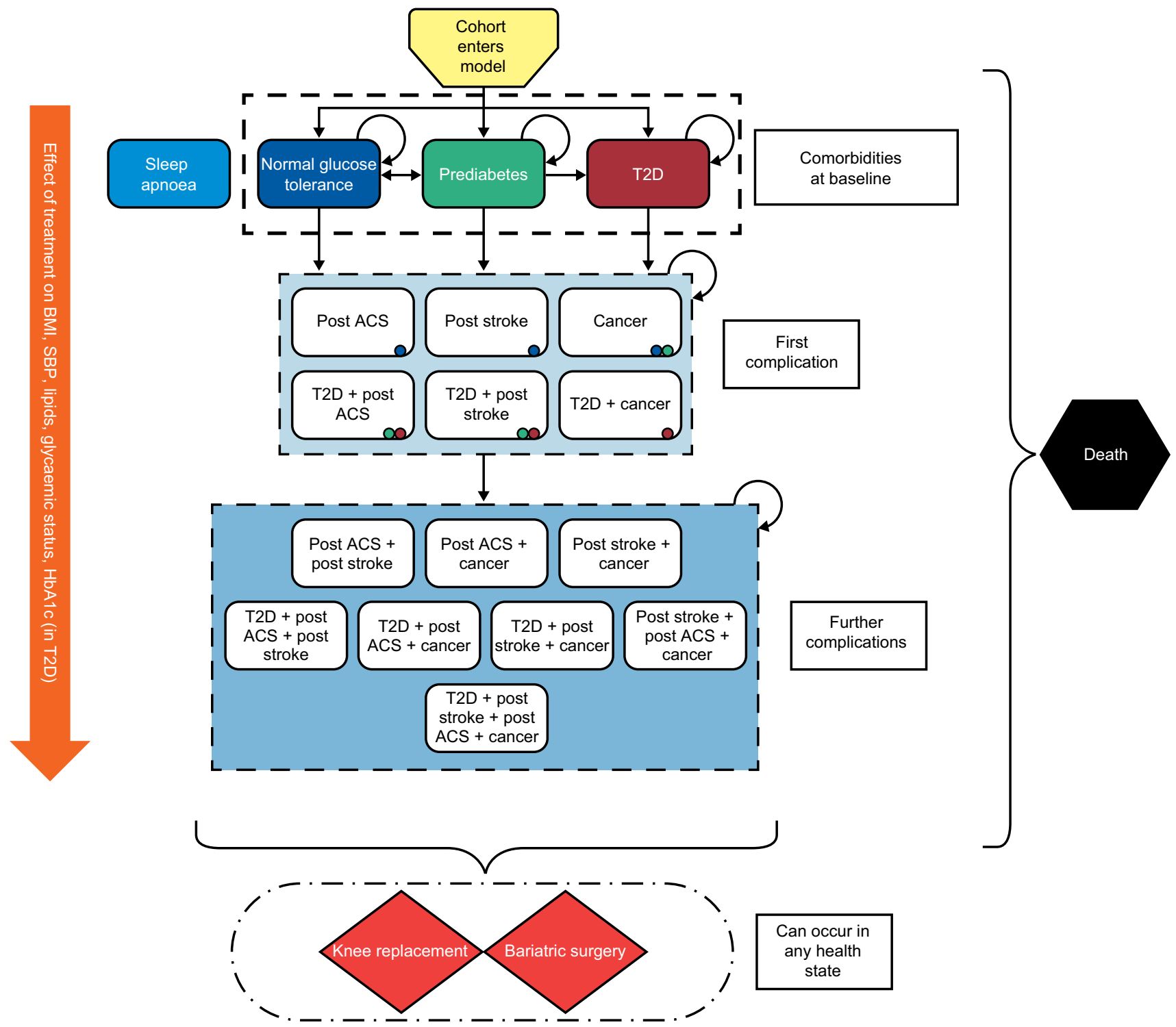

Fig. 1 Structure of the Core Obesity Model. ACS acute coronary syndrome, BMI body mass index, HbAlc glycated haemoglobin, SBP systolic blood pressure, $T 2 D$ type 2 diabetes

\subsection{External Dependent and Independent Validation Process}

The external validations were based on a three-step process encompassing the identification of suitable sources and outcomes, populating the COM with relevant inputs to predict study outcomes, and the assessment of concordance between predicted and observed outcomes.

\subsubsection{Identification of Relevant Studies}

The studies used to provide inputs during the development of the COM were used for dependent validation (Online Resource 1 , see the electronic supplementary material).
For independent validation, a systematic review [16] was consulted to identify relevant studies in normoglycaemic or prediabetic populations, whereas relevant studies for populations with T2D were sourced from the published validation of the Economic and Health Outcomes Model of Type 2 Diabetes Mellitus (ECHO-T2DM) model [10]. In a subsequent step, targeted searches were conducted via Medline and Google to identify additional studies. This version of the COM (6.1) was designed to assess the long-term costeffectiveness of weight management interventions for the primary prevention of CVD and other obesity-related complications; consequently, studies in primary prevention (i.e. those with CVD history as an exclusion criterion, or those in which the proportion of individuals with CVD was less 
than $15 \%$ ) were selected for validation (Online Resource 2). Furthermore, several criteria were used to select studies that were relevant for inclusion in the independent validation. Firstly, they were considered to have potential for replication because data on baseline characteristics and long-term trends of risk factor progression were publicly available. Furthermore, selected studies had a sufficiently long time horizon, included individuals who were overweight or obese, and reported endpoints that were identical or similar to those included in the COM.

The external independent validation of $\mathrm{CV}$ outcomes and mortality in normoglycaemic or prediabetic populations was performed using data from five randomized controlled trials (RCTs) [24-28] and four observational studies [29-32] identified in the systematic review. A further five studies allowed validation of these outcomes in populations with T2D [33-37]. Four studies from the review were selected for the validation of T2D incidence [25, 27, 38, 39], and six studies for the validation of OSA, which reported data pairs of OSA prevalence and underlying BMI levels across 21 populations [40-45]. In line with recommendations from ISPOR/SMDM, data sources that provided sufficient details (e.g. patient characteristics at baseline) to enable replication of design and progression of the study or data source were prioritized for inclusion in the validation [13].

\subsubsection{Inclusion of Relevant Endpoints}

Endpoints from relevant studies were included in the analysis only if their definitions were the same as, or similar to, those used in the COM, so that substantial mismatches could be ruled out. For studies that reported composite endpoints of interest, the feasibility was assessed of estimating the composite endpoints by adding individual endpoint components of the COM. Endpoint definitions for CVD and mortality outcomes from studies included in the independent validation and the approach taken to match these endpoints in the COM are provided in Online Resource 3.

The following clinical events were assessed to determine external validity: onset of T2D, OSA, fatal or non-fatal MI, fatal or non-fatal unstable angina, fatal or non-fatal stroke, CV mortality (a composite of fatal MI, fatal angina and fatal stroke) and all-cause mortality. For the purposes of presenting results, these events were subsequently grouped into three categories: CVD and mortality, T2D incidence, and OSA.

\subsubsection{Populating the Core Obesity Model}

For each dependent or independent validation exercise, the model was populated with the baseline clinical and demographic characteristics of the populations included in the relevant study. The inputs and assumptions applied as part of the dependent CVD and mortality validations are presented in Online Resource 4 (see the electronic supplementary material); those used for the independent validations are presented in Online Resource 5, 6 and 7.

Treatment effects and the progression of risk factors over time were replicated in the model whenever such data were reported. When risk factor progression was not published in dependent validation studies, BMI was assumed to increase by $0.1447 \mathrm{~kg} / \mathrm{m}^{2} /$ year in men and $0.1747 \mathrm{~kg} / \mathrm{m}^{2} /$ year in women [19]. A linear increase of $0.5 \mathrm{mmHg} /$ year was assumed for systolic blood pressure (SBP) in all individuals [46], but no changes in total cholesterol [47] or high-density lipoprotein cholesterol were included. In the independent validation, no progression of these risk factors was assumed. For all validations, glycated haemoglobin $(\mathrm{HbAlc})$ was varied based on a published natural-progression approach [48].

The modelled time horizon was defined to match the mean or median follow-up duration of each study, and the model was applied at a cohort size of 100 patients. For cases in which the COM (which is based on annual cycle length) was compared with studies in which follow-up duration was not an integer value, the model was applied to the upper and lower integer years and linear interpolation was used to ensure that the time frame of the prediction matched the study follow-up.

Throughout all validation exercises the default configurations of risk equations in the COM were applied, from the following sources:

T2D incidence: QDiabetes ${ }^{\circledR}$ [49]

Primary CV events in normoglycaemic and prediabetic populations: QRISK®3 [50]

Secondary CV events in normoglycaemic populations: Framingham [51]

Primary and secondary CV events in T2D populations: UK Prospective Diabetes Study (UKPDS) 82 [52]

\subsubsection{Assessment of Concordance}

Several statistical tests have been proposed for comparing model predictions with observed outcomes; however, there is no consensus on the best approach [10]. Model concordance was evaluated by assessing goodness of fit. Visual inspection of model fit was undertaken by plotting the predicted outcomes ( $y$ axis) against the observed study endpoints ( $x$ axis). A $45^{\circ}$ identity line (IL) was then plotted, reflecting a situation whereby the predicted and observed results matched perfectly. Overpredictions or underpredictions were indicated by large numbers of points above or below the IL, respectively. Additionally, an ordinary leastsquares linear regression line (OLS LRL) was fitted to the data. This had an intercept of zero to attribute more weight to the OLS LRL slope. A value for the slope markedly lower than 1.0 suggests underprediction by the model and a slope 
value greater than 1.0 suggests overprediction. Coefficient of determination $\left(R^{2}\right)$ was obtained for all validation results and quantifies how close the validation outcomes were to the OLS LRL.

A number of mean error estimates were generated as part of the validations, to provide a broad picture of areas in which model fit was poor and to quantify the magnitude of mismatch between the predicted and observed data. The estimates generated were mean absolute percentage error (MAPE), root mean squared percentage error (RMSPE), mean squared log of accuracy ratio (MSLAR) and mean squared root logit error (MSLE). If the model predicted outcomes perfectly, the measures of error would equal zero. The quality of the prediction decreased with increasing values.

\section{Results}

Full details of the outcomes from the dependent and independent validations are provided in Online Resource 8 (dependent) and Online Resource 9, 10, 11, 12 and 13 (independent) (see the electronic supplementary material); key results are discussed below.

\subsection{Dependent Validation}

\subsubsection{CVD}

External dependent validations for CVD were conducted against the Framingham Heart Study, QRISK ${ }^{\circledR} 3$ and the UKPDS 80 [50, 53, 54]. Overall, there was concordance between the outcomes predicted by the COM and the outcomes observed in the three studies (Fig. 2a; relationship between predicted and observed outcomes in each study displayed as separate data points). The results displayed a high degree of linear correlation $\left(R^{2}=0.927\right)$, and only a modest underestimation of validation outcomes (indicated by the slope of the OLS LRL $=0.859$ ).

\subsubsection{T2D}

External dependent validation of T2D incidence was conducted using the Framingham Offspring Study and QDiabetes ${ }^{\circledR}[49,55]$. Overall, there was almost $100 \%$ concordance between the observed and predicted outcomes for T2D incidence. The corresponding slope of the OLS LRL was 0.942 , and the $R^{2}$ statistic was 0.979 (Fig. 2b).

\subsubsection{OSA}

The COM uses data from the Sleep Heart Health Study [56] to predict OSA prevalence conditional on time-updated BMI; therefore, external dependent validation for OSA involved verification of consistency between the associations derived from this study and related changes in OSA prevalence following BMI variations in the COM. External dependent validation found $100 \%$ concordance between these associations, resulting in a single line when plotted (Fig. 2c).

\subsubsection{Mean Error Estimates}

A summary of the mean error rate outcomes observed from the external validations of the COM is presented in Table 1 . Overall, the mean error rates were lowest in predictions of T2D incidence, with MAPE, RMSPE, MSLAR and MSLE values of $10.6 \%, 12.3 \%, 1.5 \%$ and $0.0 \%$, respectively, compared with values of $25.4 \%, 32.9 \%$. $10.7 \%$ and $3.9 \%$, respectively, for predictions of CVD and mortality events.

\subsection{Independent Validation}

\subsubsection{CVD}

External independent validations were conducted for CVD and mortality using 14 studies, which encompassed more than 150 outcomes [24-37]. Overall, the incidence of study outcomes tended to be slightly underestimated by the COM, especially for CV mortality outcomes. The slope of the OLS LRL was estimated at 0.811 , accompanied by a linear correlation coefficient of $R^{2}=0.819$ (Fig. 3a).

Scenario analyses encompassing stratification of the overall results for CVD and mortality events by specific populations (populations with normal glucose tolerance, prediabetes or T2D), by study design (RCT or observational study) and by individual CVD outcomes (MI, stroke, unstable angina and mortality) are presented in Online Resources 11,12 and 13 (see the electronic supplementary material). The analyses suggest that the observed underprediction in the overall results may be a result of predictions made specifically in populations with T2D (Online Resource 14a), comparisons with results from RCTs (Online Resource 15a) and predictions of stroke or mortality (including all-cause mortality and CV mortality) events (Online Resource 16b and d). There was also a moderate overestimation of angina events by the model (Online Resource 16c).

\subsubsection{T2D}

External independent validations for T2D incidence were conducted using four studies [25, 27, 38, 39]. Overall, the incidence of T2D in these studies tended to be slightly underestimated by the COM (Online Resource 12). As such, the slope of the OLS LRL was estimated at 0.838 , and a high degree of linear correlation was observed $\left(R^{2}=0.968\right.$; Fig. 3b). 
Fig. 2 Observed versus predicted results from the dependent validations of a CVD and mortality outcomes, $\mathbf{b}$ T2D incidence and $\mathbf{c}$ OSA prevalence. a Linear regression analysis of observed versus predicted CVD and mortality outcomes (symbols), with the solid line representing the IL and the dashed line representing the OLS LRL and associated 95\% confidence interval (grey area) [50, 53, 54]. b Linear regression analysis of observed versus predicted T2D incidence outcomes (symbols), with the solid line representing the IL and the dashed line representing the OLS LRL $[49,55]$. c Observed OSA prevalence with increasing BMI as given in the SHHS [56]. BMI body mass index, CV cardiovascular, CVD cardiovascular disease, $I L$ identity line, $M I$ myocardial infarction, $O L S L R L$ ordinary leastsquares linear regression line, OSA, obstructive sleep apnoea, $R^{2}$ coefficient of determination, SHHS Sleep Heart Health Study, T2D type 2 diabetes, TIA transient ischaemic attack

\subsubsection{OSA}

External independent validation was based on the comparison of reported OSA prevalence score and underlying BMI levels across six studies [40-45] (Online Resource 13). The distribution of study estimates was wide but homogeneous, suggesting that the values presented in the Sleep Heart Health Study [56] provide a reasonable average of the observed data (Fig. 3c). Due to the shape of the relationship, the OLS LRL and $R^{2}$ statistics have limited value.

\subsubsection{Mean Error Estimates}

A summary of the mean error rate outcomes observed from the dependent validations of the COM is presented in Table 1. Overall, the mean error estimates generated during the independent validations were higher than those observed for the dependent validations. Furthermore, the mean error estimates were generally lowest for the predictions of CVD and mortality events, with MAPE, RMSPE, MSLAR and MSLE values of $55.2 \%, 93.0 \%, 31.2 \%$ and $20.0 \%$, respectively.

\section{Discussion}

The ISPOR/SMDM guidelines highlight the need for transparency and validation to ensure that economic models are fit for purpose and that their outputs can be interpreted with confidence [13]; however, the majority of published economic models in obesity have not been subject to external validation [16]. In this study, we focussed on the results of the external dependent and independent validations of the COM, which build on the face validity and verification that had been conducted throughout model development. Face validity was evaluated using clinical expert feedback and discussions arising from presentation of the model structure at conferences. Verification was performed through reproduction of computational examples of the published risk
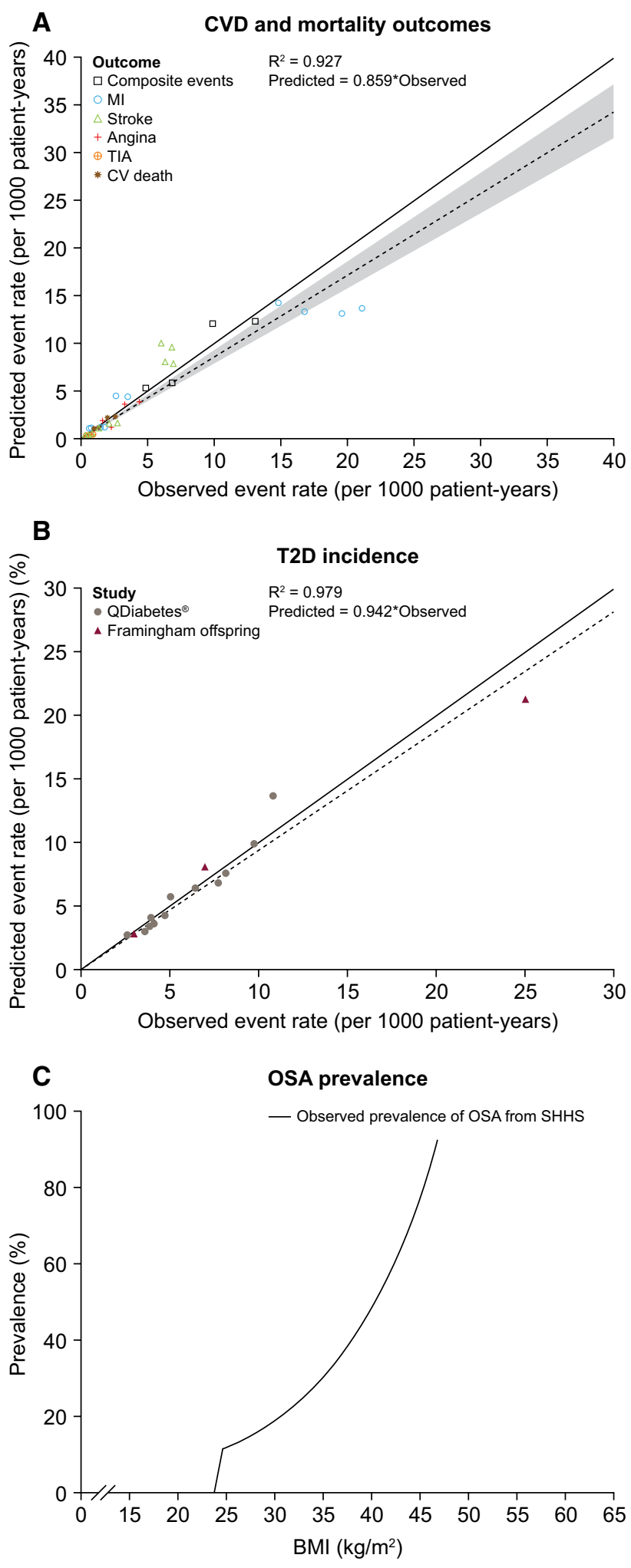

equations or via consistency checks with a double-programming approach, which demonstrated a $100 \%$ correspondence between distinct model implementations, thereby confirming an error-free use of risk equations within the COM. The use 
Table 1 Summary of external validation mean error values

\begin{tabular}{lllllll}
\hline & MAPE (\%) & RMSPE (\%) & MSLAR (\%) & MSLE (\%) & $R^{2}$ & Slope \\
\hline Dependent validation & & & & & & \\
CVD and mortality & 25.4 & 32.9 & 10.7 & 3.9 & 0.927 & 0.859 \\
T2D incidence & 10.6 & 12.3 & 1.5 & 0.0 & 0.979 & 0.942 \\
OSA & NA & NA & NA & NA & NA & NA \\
Independent validation & & & & & & \\
CVD and mortality & 55.2 & 93.0 & 31.2 & 20.0 & 0.819 & 0.811 \\
T2D incidence & 73.9 & 164.1 & 55.9 & 0.3 & 0.968 & 0.838 \\
OSA & 129.4 & 213.2 & 173.4 & 5.1 & -0.188 & NA \\
\hline
\end{tabular}

$C V D$ cardiovascular disease, MAPE mean absolute percentage error, MSLAR mean squared log of accuracy ratio, MSLE mean squared root logit error, $N A$ not applicable, $O S A$ obstructive sleep apnoea, $R^{2}$ coefficient of determination, $R M S P E$ root mean squared percentage error, $T 2 D$ type 2 diabetes of prospective validation was not feasible at the time of this analysis; a cross-validation analysis is planned as a future publication.

Having performed both dependent and independent external validation, our findings suggested that the risk equations upon which the COM is based generate reasonable predictions, and the model is likely to be generalizable to other settings, including different countries and patient populations. The concordance between model predictions and the selected studies also suggests that, based on mean results, the COM predicts event rates with reasonable accuracy.

Independent validations of the COM showed good concordance with the results of relevant studies, as indicated by high coefficients of determination; however, there was a degree of underprediction for CVD and mortality outcomes, and for T2D incidence. Scenario analyses revealed that this overall underprediction of CV events by the COM may stem from predictions made in populations with T2D, comparisons made with outcomes from RCTs or predictions of stroke and mortality. Furthermore, the underprediction observed for all-cause mortality demonstrates the continuing refinement required for economic models, and highlights the incomplete understanding of the relationship between BMI and mortality. The finding that $\mathrm{CV}$ death was also underestimated suggests that there may be unknown risk factors that result in the underestimation of mortality in individuals with obesity. Furthermore, the underprediction observed during comparisons with the results from RCTs may have been due to the specific baseline characteristics of the study populations included in these trials. Indeed, many of the study populations did not include individuals with obesity, and some also included study populations with restricted lipid profiles [27].

The underprediction observed may have also resulted from the use of mean inputs into the COM, which are not fully representative of the heterogeneous populations in the real world [57]. However, it should be noted that alternative, probabilistic modelling approaches that assume sampling with distributions typically tend to predict higher event rates than use of mean inputs. Furthermore, the underprediction of CVD outcomes in both the dependent and independent validations may have occurred because of the absence of CVD at baseline in the COM, whereas individuals with CVD at baseline were included in some of the comparator studies, comprising up to $15 \%$ of the population. It should be noted that some of the observed underpredictions may be mitigated when using the COM to generate incremental outcomes, because the underprediction would be present in both the intervention and comparator arms provided that it is independent of treatment effects. However, the underprediction of some CVD outcomes may extend to events that would be prevented by treatment, resulting in treatment efficacy being underestimated and thus leading to higher incremental cost-effectiveness ratios than those generated by a model that does not show the same tendency for underprediction.

These analyses were performed in accordance with best-practice recommendations for the validation of costeffectiveness models [12, 13], and the concordance between external validations adds weight to our conclusions. The findings were based on several different observations encompassing goodness of fit, in addition to visual inspection, $R^{2}$ statistics, match between the IL and the OLS LRL, and error terms. Furthermore, the independent external validation was performed across a broad range of studies, spanning 193 validation endpoints (162 CV and mortality outcomes, ten T2D incidence outcomes and 21 OSA outcomes). These studies also comprised a mixture of RCTs and observational studies conducted across different countries and across different time periods, improving confidence that the concordance observed is generalizable across multiple settings. Although the concordance of these observations is improved by inclusion of numerous studies and endpoints, it should also be noted that the disparities between the populations included in RCTs and those in observational studies may 
Fig. 3 Observed versus predicted results from the independent validations of a CVD and mortality outcomes, b T2D incidence and $\mathbf{c}$ OSA prevalence. a Linear regression analyses of observed versus predicted CVD and mortality outcomes (symbols), with the solid line representing the IL and the dashed line representing the OLS LRL and associated 95\% confidence interval (grey area) [24-37]. b Linear regression analysis of observed versus predicted T2D incidence (symbols), with the solid line representing the IL and the dashed line representing the OLS LRL and associated 95\% confidence interval (grey area) [25, 27, 38, 39]. c Observed OSA prevalence with increasing BMI as given in the SHHS (solid line) compared with observed outcomes stratified by BMI group (symbols) [40-45]. ACM all-cause mortality, BMI body mass index, CV cardiovascular, CVD cardiovascular disease, $I L$ identity line, $M I$ myocardial infarction, $O L S L R L$ ordinary least-squares linear regression line, OSA obstructive sleep apnoea, $R^{2}$ coefficient of determination, SHHS Sleep Heart Health Study, T2D type 2 diabetes

have contributed to some of the overestimation and underestimation by the model.

Given the nature of validation analyses, there were some limitations to our study. Some of the baseline characteristics required to populate the COM (SBP, total cholesterol, high-density lipoprotein cholesterol, proportion of individuals who smoke) and measures of risk factor evolution over time (e.g. natural increase in weight) were not available for all comparator studies, and use of substitute values may have introduced some uncertainty by creating mismatches between the simulated cohort and the study participants. This risk was mitigated by carefully selecting the studies used to populate these missing values, ensuring that they had a similar population to the other studies included in the validation. These mismatches between simulated and study populations may have been particularly apparent in comparisons with RCTs because of their more stringent inclusion criteria and follow-up periods, compared with those of observational studies. Indeed, the scenario analysis in which CVD outcomes were stratified by study design showed a larger underprediction for comparisons with RCTs than with observational studies, suggesting that the predictions may have been closer to observed values if RCTs were omitted from the independent validation. A further limitation that is common to economic models is the fact that the accuracy of predictions depends on the quality and scope of the data available at the time of development. A key area of future development will be the identification of additional correction factors, to mitigate the possibility that known and/or unknown factors underlie the observed underprediction of mortality. Additionally, our model was affected by a lack of available risk equations that encompass changes in BMI as a covariate, which meant that we could not fully assess residual risk of BMI history.

Our results indicate the accuracy of predictions made using a cohort state-transition model of obesity; a comparison of the accuracy of different modelling methods in this disease area would be of value in the future, but falls outside
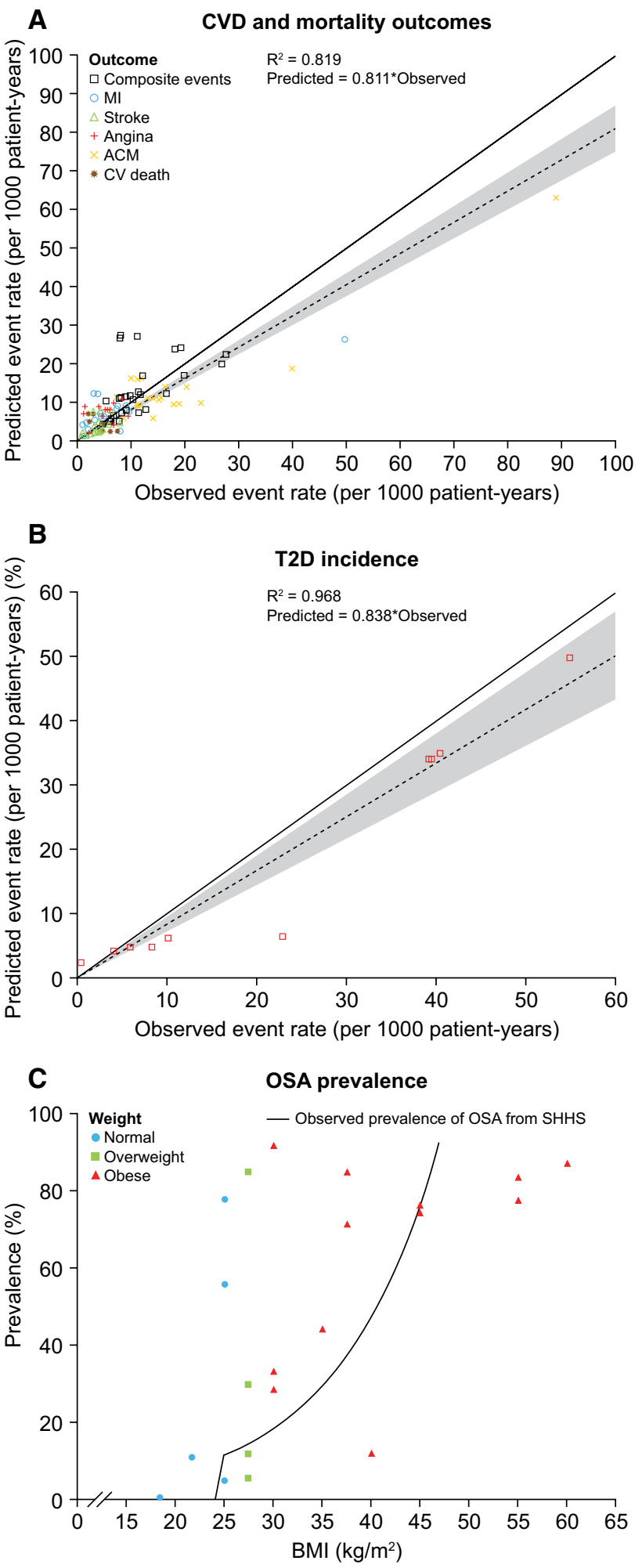

the scope of this analysis. We also focused on external validation of event risk predictions made by the COM rather than comparisons of observed and predicted costs. Such analyses are complex to conduct [13], and would require a separate publication to explore fully. 


\section{Conclusion}

These external validation analyses revealed that the predictions generated by the risk equations used in the COM show good concordance both with the studies used to develop the model and with other studies not included in the model. Despite this concordance, the predictions made by the COM can be improved; the ability of this model to capture the impact of existing CV risk, as well as mortality, are key areas for future refinement. Taken together, these results should increase confidence in the estimates derived from the COM and reduce uncertainty associated with analyses using this model.

Acknowledgements The authors acknowledge the medical writing assistance of PharmaGenesis Oxford Central, which was funded by Novo Nordisk A/S.

Author Contributions All authors were involved in the design of the work as well as the analysis and interpretation of the data. PM and VF performed the validations. All authors reviewed and revised the manuscript for important intellectual content.

\section{Compliance with Ethical Standards}

Conflict of interest Sandra Lopes and Pierre Johansen are employees of and shareholders in Novo Nordisk A/S. IQVIA, the employer of Mark Lamotte and Anna-Maria Olivieri, received consulting fees from Novo Nordisk A/S for this study. Health Economics and Outcomes Research Ltd., the employer of Phil McEwan and Volker Foos, received consulting fees from Novo Nordisk A/S for this study.

Funding Funding for this study was provided by Novo Nordisk A/S

Availability of Data and Material All data generated or analysed during this study are included in this published article (and its supplementary information files).

Code availability Not applicable.

Open Access This article is licensed under a Creative Commons Attribution-NonCommercial 4.0 International License, which permits any non-commercial use, sharing, adaptation, distribution and reproduction in any medium or format, as long as you give appropriate credit to the original author(s) and the source, provide a link to the Creative Commons licence, and indicate if changes were made. The images or other third party material in this article are included in the article's Creative Commons licence, unless indicated otherwise in a credit line to the material. If material is not included in the article's Creative Commons licence and your intended use is not permitted by statutory regulation or exceeds the permitted use, you will need to obtain permission directly from the copyright holder. To view a copy of this licence, visit http://creativecommons.org/licenses/by-nc/4.0/.

\section{References}

1. World Health Organization. Overweight and obesity. 16 February 2018. https://www.who.int/news-room/fact-sheets/detail/obesityand-overweight. Accessed 10 June 2019.
2. Li C, Ford ES, Zhao G, Croft JB, Balluz LS, Mokdad AH. Prevalence of self-reported clinically diagnosed sleep apnea according to obesity status in men and women: National Health and Nutrition Examination Survey, 2005-2006. Prev Med. 2010;51(1):18 23. https://doi.org/10.1016/j.ypmed.2010.03.016.

3. Khan SS, Ning H, Wilkins JT, Allen N, Carnethon M, Berry JD, et al. Association of body mass index with lifetime risk of cardiovascular disease and compression of morbidity. JAMA Cardiol. 2018;3(4):280-7. https://doi.org/10.1001/jamacardio.2018.0022.

4. Must A, Spadano J, Coakley EH, Field AE, Colditz G, Dietz WH. The disease burden associated with overweight and obesity. JAMA. 1999;282(16):1523-9. https://doi.org/10.1001/ jama.282.16.1523.

5. Health matters: obesity and the food environment. 2017. Available from: https://www.gov.uk/government/publications/health-matte rs-obesity-and-the-food-environment/health-matters-obesity-andthe-food-environment--2. Accessed 25 October 2019.

6. Ul-Haq Z, Mackay DF, Fenwick E, Pell JP. Meta-analysis of the association between body mass index and health-related quality of life among adults, assessed by the SF-36. Obesity (Silver Spring). 2013;21(3):E322-E327327. https://doi.org/10.1002/oby.20107.

7. Counterweight Project $T$. The impact of obesity on drug prescribing in primary care. Br J Gen Pract. 2005;55(519):743-9.

8. Herrett E, Gallagher AM, Bhaskaran K, Forbes H, Mathur R, van Staa T, et al. Data resource profile: clinical practice research Datalink (CPRD). Int J Epidemiol. 2015;44(3):827-36. https:// doi.org/10.1093/ije/dyv098.

9. Hoque ME, Mannan M, Long KZ, Al MA. Economic burden of underweight and overweight among adults in the AsiaPacific region: a systematic review. Trop Med Int Health. 2016;21(4):458-69. https://doi.org/10.1111/tmi.12679.

10. Willis M, Johansen P, Nilsson A, Asseburg C. Validation of the economic and health outcomes model of type 2 diabetes mellitus (ECHO-T2DM). Pharmacoeconomics. 2017;35(3):375-96. https ://doi.org/10.1007/s40273-016-0471-3.

11. American Diabetes Association Consensus P. Guidelines for computer modeling of diabetes and its complications. Diabetes Care. 2004;27(9):2262-5. https://doi.org/10.2337/diacare.27.9.2262.

12. Caro JJ, Briggs AH, Siebert U, Kuntz KM, Force I-SMGRPT. Modeling good research practices-overview: a report of the ISPOR-SMDM Modeling Good Research Practices Task Force1. Value Health. 2012;15(6):796-803. https://doi.org/10.1016/j. jval.2012.06.012.

13. Eddy DM, Hollingworth W, Caro JJ, Tsevat J, McDonald KM, Wong JB, et al. Model transparency and validation: a report of the ISPOR-SMDM Modeling Good Research Practices Task Force-7. Med Decis Mak. 2012;32(5):733-43. https://doi. org/10.1177/0272989X12454579.

14. Kent S, Becker F, Feenstra T, Tran-Duy A, Schlackow I, Tew $\mathrm{M}$, et al. The challenge of transparency and validation in health economic decision modelling: a view from Mount Hood. Pharmacoeconomics. 2019;37(11):1305-12. https://doi.org/10.1007/ s40273-019-00825-1.

15. Palmer AJ, Si L, Tew M, Hua X, Willis MS, Asseburg C, et al. Computer modeling of diabetes and its transparency: a report on the Eighth Mount Hood Challenge. Value Health. 2018;21(6):724-31. https://doi.org/10.1016/j.jval.2018.02.002.

16. Schwander B, Nuijten M, Hiligsmann M, Evers S. Event simulation and external validation applied in published health economic models for obesity: a systematic review. Expert Rev Pharmacoecon Outcomes Res. 2018;18(5):529-41. https://doi. org/10.1080/14737167.2018.1501680.

17. De Francesco M, Lopes S, Meincke HH, Vega-Hernandez V, Lamotte M, Lean MEJ. Core Obesity Model to assess the costeffectiveness of weight management interventions [poster]. In: Presented at the International Society for Pharmacoeconomics 
and Outcomes Research (ISPOR) Europe 2018, 10-14 November 2018, Barcelona, Spain.

18. World Health Organization. Obesity: preventing and managing the global epidemic. Report Of A WHO Consultation. 2000. https:// www.who.int/nutrition/publications/obesity/WHO_TRS_894/en/. Accessed 14 Oct 2019.

19. Ara R, Blake L, Gray L, et al. What is the clinical effectiveness and cost-effectiveness of using drugs in treating obese patients in primary care? A systematic review. Health Technol Assess. 2012;16(5):1-195 (iii-xiv).

20. Ara R, Blake L. PSY36 modelling the cost-effectiveness of orlistat as a treatment for obesity in primary care. Value Health. 2011;14(7):A416-A417417. https://doi.org/10.1016/j. jval.2011.08.1008.

21. Lewis L, Taylor M, Broom J, Johnston KL. The cost-effectiveness of the LighterLife weight management programme as an intervention for obesity in England. Clin Obes. 2014;4(3):180-8. https:// doi.org/10.1111/cob.12060.

22. National Institute for Health and Care Excellence. NG28. Type 2 diabetes in adults. Appendix F. 2015. https://www.nice.org.uk/ guidance/ng28/evidence/appendix-f-full-health-economicsreport -pdf-2185320355. Accessed 18 May 2020.

23. Willis M, Fridhammar A, Gundgaard J, Nilsson A, Johansen P. Comparing the cohort and micro-simulation modeling approaches in cost-effectiveness modeling of type 2 diabetes mellitus: a case study of the IHE Diabetes Cohort Model and the Economics and Health Outcomes Model of T2DM. Pharmacoeconomics. 2020. https://doi.org/10.1007/s40273-020-00922-6.

24. Wright JT Jr, Williamson JD, Whelton PK, et al. A randomized trial of intensive versus standard blood-pressure control. N Engl J Med. 2015;373(22):2103-16.

25. Dahlof B, Sever PS, Poulter NR, Wedel H, Beevers DG, Caulfield $\mathrm{M}$, et al. Prevention of cardiovascular events with an antihypertensive regimen of amlodipine adding perindopril as required versus atenolol adding bendroflumethiazide as required, in the AngloScandinavian Cardiac Outcomes Trial-Blood Pressure Lowering Arm (ASCOT-BPLA): a multicentre randomised controlled trial. Lancet. 2005;366(9489):895-906. https://doi.org/10.1016/S0140 -6736(05)67185-1.

26. Estruch R, Ros E, Salas-Salvado J, Covas MI, Corella D, Aros F, et al. Primary prevention of cardiovascular disease with a Mediterranean diet. N Engl J Med. 2013;368(14):1279-90. https://doi. org/10.1056/NEJMoa1200303.

27. Glynn RJ, Koenig W, Nordestgaard BG, Shepherd J, Ridker PM. Rosuvastatin for primary prevention in older persons with elevated C-reactive protein and low to average low-density lipoprotein cholesterol levels: exploratory analysis of a randomized trial. Ann Intern Med. 2010;152(8):488-96. https://doi.org/10.7326/00034819-152-8201004200-00005 (W174).

28. Yusuf S, Lonn E, Pais P, Bosch J, Lopez-Jaramillo P, Zhu J, et al. Blood-pressure and cholesterol lowering in persons without cardiovascular disease. N Engl J Med. 2016;374(21):2032-43. https ://doi.org/10.1056/NEJMoa1600177.

29. Anand SS, Dagenais GR, Mohan V, Diaz R, Probstfield J, Freeman $\mathrm{R}$, et al. Glucose levels are associated with cardiovascular disease and death in an international cohort of normal glycaemic and dysglycaemic men and women: the EpiDREAM cohort study. Eur J Prev Cardiol. 2012;19(4):755-64. https://doi.org/10.1177/17418 26711409327.

30. Engstrom G, Hedblad B, Stavenow L, Jonsson S, Lind P, Janzon L, et al. Incidence of obesity-associated cardiovascular disease is related to inflammation-sensitive plasma proteins: a population-based cohort study. Arterioscler Thromb Vasc Biol. 2004;24(8):1498-502. https://doi.org/10.1161/01.ATV.00001 34293.31512.be.
31. Kurth T, Gaziano JM, Berger K, Kase CS, Rexrode KM, Cook $\mathrm{NR}$, et al. Body mass index and the risk of stroke in men. Arch Intern Med. 2002;162(22):2557-622. https://doi.org/10.1001/ archinte.162.22.2557.

32. Thomsen M, Nordestgaard BG. Myocardial infarction and ischemic heart disease in overweight and obesity with and without metabolic syndrome. JAMA Intern Med. 2014;174(1):15-22. https://doi.org/10.1001/jamainternmed.2013.10522.

33. Look AHEAD Research Group. Cardiovascular effects of intensive lifestyle intervention in type 2 diabetes. N Engl J Med. 2013;369(2):145-54

34. UK Prospective Diabetes Study (UKPDS) Group. Intensive bloodglucose control with sulphonylureas or insulin compared with conventional treatment and risk of complications in patients with type 2 diabetes (UKPDS 33). Lancet. 1998;352(9131):837-53.

35. Colhoun HM, Betteridge DJ, Durrington PN, Hitman GA, Neil HA, Livingstone SJ, et al. Primary prevention of cardiovascular disease with atorvastatin in type 2 diabetes in the Collaborative Atorvastatin Diabetes Study (CARDS): multicentre randomised placebo-controlled trial. Lancet. 2004;364(9435):685-96. https:// doi.org/10.1016/S0140-6736(04)16895-5.

36. Knopp RH, d'Emden M, Smilde JG, Pocock SJ. Efficacy and safety of atorvastatin in the prevention of cardiovascular end points in subjects with type 2 diabetes: the Atorvastatin Study for Prevention of Coronary Heart Disease Endpoints in noninsulin-dependent diabetes mellitus (ASPEN). Diabetes Care. 2006;29(7):1478-85. https://doi.org/10.2337/dc05-2415.

37. Sasaki A, Uehara M, Horiuchi N, Hasegawa K, Shimizu T. A 15 year follow-up study of patients with non-insulin dependent diabetes mellitus (NIDDM) in Osaka, Japan. Long-term prognosis and causes of death. Diabetes Res Clin Pract. 1996;34(1):47-55. https://doi.org/10.1016/s0168-8227(96)01329-0.

38. Ramachandran A, Snehalatha C, Mary S, Mukesh B, Bhaskar AD, Vijay V, et al. The Indian Diabetes Prevention Programme shows that lifestyle modification and metformin prevent type 2 diabetes in Asian Indian subjects with impaired glucose tolerance (IDPP1). Diabetologia. 2006;49(2):289-97. https://doi.org/10.1007/ s00125-005-0097-z.

39. Tuomilehto J, Lindstrom J, Eriksson JG, Valle TT, Hamalainen $\mathrm{H}$, Ilanne-Parikka P, et al. Prevention of type 2 diabetes mellitus by changes in lifestyle among subjects with impaired glucose tolerance. N Engl J Med. 2001;344(18):1343-50. https://doi. org/10.1056/NEJM200105033441801.

40. Wosu AC, Vélez JC, Barbosa C, et al. The relationship between high risk for obstructive sleep apnea and general and central obesity: findings from a sample of Chilean college students. ISRN Obes. 2014;2014:871681.

41. O'Keeffe T, Patterson EJ. Evidence supporting routine polysomnography before bariatric surgery. Obes Surg. 2004;14(1):23-6.

42. Dacal Quintas R, Tumbeiro Novoa M, Alves Perez MT, et al. Obstructive sleep apnea in normal weight patients: characteristics and comparison with overweight and obese patients. Arch Bronconeumol. 2013;49(12):513-7.

43. Chen X, Pensuksan WC, Lohsoonthorn V, Lertmaharit S, Gelaye B, Williams MA. Obstructive sleep apnea and multiple anthropometric indices of general obesity and abdominal obesity among young adults. Int J Soc Sci Stud. 2014;2(3):89-99.

44. Lopez PP, Stefan B, Schulman CI, Byers PM. Prevalence of sleep apnea in morbidly obese patients who presented for weight loss surgery evaluation: more evidence for routine screening for obstructive sleep apnea before weight loss surgery. Am Surg. 2008;74(9):834-8.

45. Wall H, Smith C, Hubbard R. Body mass index and obstructive sleep apnoea in the UK: a cross-sectional study of the over-50s. Prim Care Respir J. 2012;21(4):371-6. https://doi.org/10.4104/ pcrj.2012.00053. 
46. Franklin SS, Gustin WT, Wong ND, Larson MG, Weber MA, Kannel WB, et al. Hemodynamic patterns of age-related changes in blood pressure. The Framingham Heart Study. Circulation. 1997;96(1):308-15. https://doi.org/10.1161/01.cir.96.1.308.

47. Arbeev KG, Ukraintseva SV, Kulminski AM, Akushevich I, Arbeeva LS, Culminskaya IV, et al. Effect of the APOE polymorphism and age trajectories of physiological variables on mortality: application of genetic stochastic process model of aging. Scientifica (Cairo). 2012. https://doi.org/10.6064/2012/568628.

48. Clarke PM, Gray AM, Briggs A, Farmer AJ, Fenn P, Stevens RJ, et al. A model to estimate the lifetime health outcomes of patients with type 2 diabetes: the United Kingdom Prospective Diabetes Study (UKPDS) Outcomes Model (UKPDS no. 68). Diabetologia. 2004;47(10):1747-59. https://doi.org/10.1007/s0012 5-004-1527-z.

49. Hippisley-Cox J, Coupland C. Development and validation of QDiabetes-2018 risk prediction algorithm to estimate future risk of type 2 diabetes: cohort study. BMJ. 2017;359:j5019. https:// doi.org/10.1136/bmj.j5019.

50. Hippisley-Cox J, Coupland C, Brindle P. Development and validation of QRISK3 risk prediction algorithms to estimate future risk of cardiovascular disease: prospective cohort study. BMJ. 2017;357:j2099. https://doi.org/10.1136/bmj.j2099.

51. D'Agostino RB, Russell MW, Huse DM, Ellison RC, Silbershatz $\mathrm{H}$, Wilson $\mathrm{PW}$, et al. Primary and subsequent coronary risk appraisal: new results from the Framingham study. Am Heart J. 2000;139(2 Pt 1):272-81. https://doi.org/10.1067/mhj.2000.96469
52. Hayes AJ, Leal J, Gray AM, Holman RR, Clarke PM. UKPDS outcomes model 2: a new version of a model to simulate lifetime health outcomes of patients with type 2 diabetes mellitus using data from the 30 year United Kingdom Prospective Diabetes Study: UKPDS 82. Diabetologia. 2013;56(9):1925-33. https://doi. org/10.1007/s00125-013-2940-y.

53. D'Agostino RB Sr, Vasan RS, Pencina MJ, Wolf PA, Cobain M, Massaro JM, et al. General cardiovascular risk profile for use in primary care: the Framingham Heart Study. Circulation. 2008;117(6):743-53. https://doi.org/10.1161/CIRCULATIO NAHA.107.699579.

54. Holman RR, Paul SK, Bethel MA, Matthews DR, Neil HA. 10-year follow-up of intensive glucose control in type 2 diabetes. N Engl J Med. 2008;359(15):1577-89. https://doi.org/10.1056/ NEJMoa0806470.

55. Wilson PW, Meigs JB, Sullivan L, Fox CS, Nathan DM, D'Agostino RB Sr. Prediction of incident diabetes mellitus in middle-aged adults: the Framingham Offspring Study. Arch Intern Med. 2007;167(10):1068-74. https://doi.org/10.1001/archi nte.167.10.1068.

56. Young T, Shahar E, Nieto FJ, Redline S, Newman AB, Gottlieb DJ, et al. Predictors of sleep-disordered breathing in communitydwelling adults: the Sleep Heart Health Study. Arch Intern Med. 2002;162(8):893-900. https://doi.org/10.1001/archinte.162.8.893.

57. Foos V, McEwan P, Lamotte M. Implications of introducing patient heterogeneity in cost effectiveness modeling. Value Health. 2016;19(7):A371. 